\title{
Join and Meet Operations for Interval-Valued Complex Fuzzy Logic
}

\author{
Sarah Greenfield \\ Centre for Computational Intelligence \\ and DMU Interdisciplinary Group in \\ Intelligent Transport Systems (DIGITS) \\ School of Computer Science \\ and Informatics \\ De Montfort University \\ Leicester LE1 9BH, UK. \\ Email: s.greenfield@dmu.ac.uk
}

\author{
Francisco Chiclana \\ Centre for Computational Intelligence \\ School of Computer Science \\ and Informatics \\ De Montfort University \\ Leicester LE1 9BH, UK. \\ Email: chiclana@dmu.ac.uk
}

\author{
Scott Dick \\ Department of Electrical \\ and Computer Engineering \\ University of Alberta \\ Edmonton \\ Alberta, Canada. \\ Email: sdick@ualberta.ca
}

\begin{abstract}
Interval-valued complex fuzzy logic is able to handle scenarios where both seasonality and uncertainty feature. The interval-valued complex fuzzy set is defined, and the intervalvalued complex fuzzy inferencing system outlined. Highly pertinent to complex fuzzy logic operations is the concept of rotational invariance, which is an intuitive and desirable characteristic. Interval-valued complex fuzzy logic is driven by interval-valued join and meet operations. Four pairs of alternative algorithms for these operations are specified; three pairs possesses the attribute of rotational invariance, whereas the other pair lacks this characteristic.
\end{abstract}

\section{INTRODUCTION}

In the 50 years since Zadeh [27] introduced the type-1 fuzzy set, the concept has undergone further development. In 1975, Zadeh went on to define the type-2 fuzzy set [28], [29], [30]. Complex fuzzy sets, applicable to the modelling of periodic phenomena, are a relatively recent development in fuzzy set theory, proposed by Ramot et al. in 2002 [22].

Data is frequently characterised by both uncertainty and seasonality. Uncertainty in the data may derive from factors such as corruption, or from elements being missing. Seasonality, whereby the data has an underlying repeating, cyclical pattern, is a very common characteristic of data. Examples of seasonality are: 1) Temperatures tend to be higher in the summer and lower in the winter; 2) There is more traffic on the roads during the rush hours.

Ramot et al. [22, page 171] present complex fuzzy sets as type-1 fuzzy sets extended by an additional phase term which permits them to intuitively represent fuzziness in time-series applications. According to Dick [5], “... the proper role for a complex fuzzy set is a remarkably efficient representation of approximately periodic phenomena, and as the underlying mathematical foundation of regularity."

At the beginning of the millennium, potential applications of complex fuzzy logic were envisaged as including the analysis of solar activity as measured by the recorded number of sunspots [22], signal processing [22], stock trading on the New York Stock Exchange [21], and prediction of voter turnout in elections [21]. Two of these potential applications have since been realised with research reported on the prediction of annual sunspot numbers [18], and financial time-series forecasting [16]. Additionally, successful applications have been developed in relation to prediction of solar power output [26], Australian bushfire danger rating (involving multi-periodic factors) [18], and multi-variate time-series forecasting of hotel occupancy and revenue generated in Victoria, Australia [25], monthly flour prices on commodity exchanges in the USA [25], and precipitation in Tennessee, USA [25]. Complex fuzzy logic is proving to be a significant and growing topic of research and application.

Type-2 fuzzy sets [28], [29], [30] are an extension of type1 fuzzy sets in which the sets' membership grades are themselves type-1 fuzzy sets. They respond to a major shortcoming of type-1 fuzzy sets by offering a conceptual scheme within which the effects of uncertainties in fuzzy inferencing may be modelled and minimised [20, page 117].

Type-2 fuzzy logic, well-suited to reasoning under uncertainty [10], [7], and complex fuzzy logic, pertinent to inferencing with seasonal data, have so far been developed independently, but there is no reason why they may not be combined into type-2 complex fuzzy logic [8], able to deal with both uncertainty and seasonality, to allow a more subtle and faithful treatment of data, leading to more precision in its analysis.

Type- 2 fuzzy sets take two forms, generalised, and the simpler interval. Interval type- 2 fuzzy sets have increasingly been used in applications [11], [15], [2], [6], [14], [24], [13], [23] as they offer a more sophisticated model of uncertainty than their type-1 counterparts [17], whilst lacking the computational complexity of the generalised type-2 fuzzy set [20], [12].

Another form of fuzzy set is the interval-valued fuzzy set, which is regarded as equivalent to the interval type-2 fuzzy set [3, Definition 1, page 217]. In [9] the concept of the Interval-Valued Complex Fuzzy Set (IVCFS) was introduced, a combination of interval-valued fuzzy sets and complex fuzzy sets. The IVCFS may be thought of as an adaption of complex fuzzy sets such that the membership function assigns each 
point of the domain to an interval.

The research presented in this paper builds upon that of [9], in which the operations constituting the Interval-Valued Complex Fuzzy Inferencing System (IVCFIS) were outlined. This paper's particular emphasis is on the join and meet operations forming the core of the IVCFIS.

In the next section, the IVCFS is defined, the IVCFIS discussed, and the concept of rotational invariance presented. Following that, in Section III, algorithms are presented for the interval-valued complex join and meet operations driving the IVCFIS. Section IV concludes the paper.

\section{INTERVAL-VALUED COMPLEX FUZZY LOGIC}

\section{A. Interval-Valued Complex Fuzzy Sets: Definitions}

The definition for the complex fuzzy set may be adapted so that the membership function assigns each point on the domain to an interval, giving the IVCFS. Before formally defining the IVCFS, we define concept of the dot product set.

Definition 1 (Dot Product Set). Given two sets $A$ and $B$ and an operator such that given $a \in A$ and $b \in B$ the element $a \cdot b$ exists, the set $A \cdot B$ can be defined:

$$
A \cdot B=\{a \cdot b ; a \in A \wedge b \in B\} .
$$

Definition 2 (Interval-Valued Complex Fuzzy Set). An interval-valued complex fuzzy set over a universe of discourse $U$ is defined by a membership function

$$
\begin{gathered}
\mu_{\bar{S}}: U \rightarrow I^{[0,1]} \cdot \dot{D} \\
\mu_{\bar{S}}(x)=r_{\bar{S}}(x) \cdot e^{j \omega_{\bar{S}}(x)}
\end{gathered}
$$

where $I^{[0,1]}$ is the set of all closed subintervals of $[0,1], \dot{D}$ is the frontier or boundary set of the unit disc $D \subset \mathbb{R} \times \mathbb{R}$, $r_{\bar{S}}(x) \in I^{[0,1]}$ is the interval-valued membership magnitude, $j=\sqrt{-1}$, and $\omega_{\bar{S}}(x)$ is the membership phase. Each element of the universe of discourse $U$ is thus associated with a set of complex numbers with moduli in a subinterval of [0,1] representing the range of possible truth-values associated with that element's membership in the complex fuzzy set, and a real-valued phase.

Figure 1(a) shows the conventional 2-D representation of the time series consisting of sunspot numbers observed on a monthly basis [1]. Figure 1(b) shows these sunspot data displayed as a 3-D complex fuzzy set. In an interval valued complex fuzzy set, each point on the domain is mapped to an interval as opposed to a crisp number. Graphically, the 3D interval-valued complex fuzzy set forms a band of points of global variable width and constant local phase within the complex plane unit circle.

Definition 3 (Membership Interval). The interval valued membership magnitude as used in Definition 2 will be referred to as the membership interval.

Definition 4 (Vertical Slice). A vertical slice of an intervalvalued complex fuzzy set is a line which intersects the $x$-axis

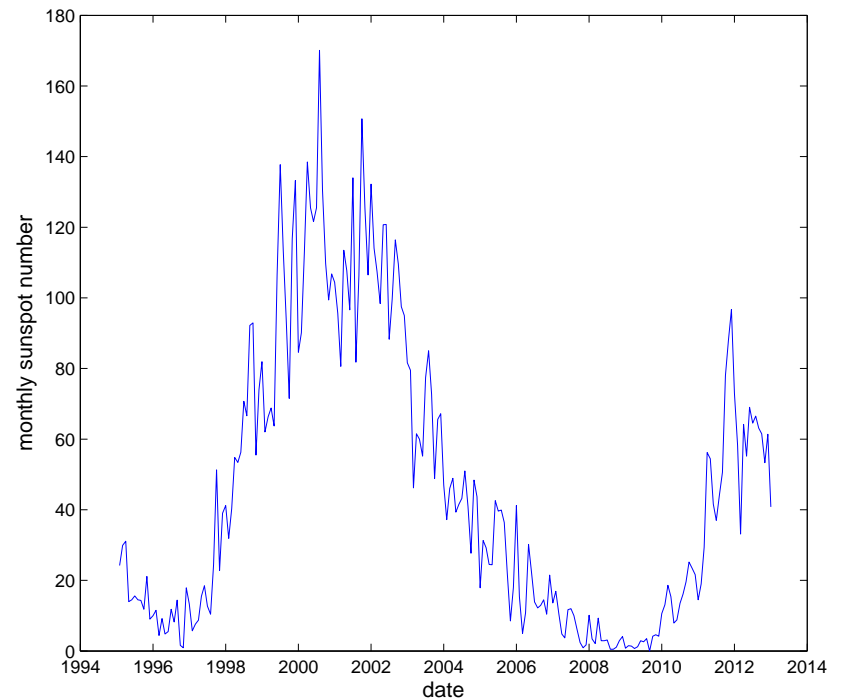

(a) Conventional representation of the sunspot data.

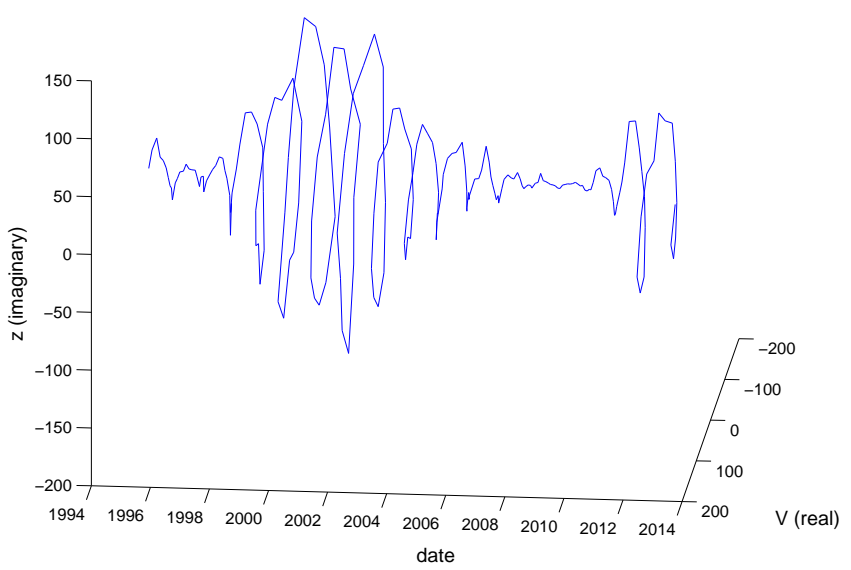

(b) Representation of the sunspot data as a complex fuzzy set in which the phase is determined by the month of observation. The modulus is greater than 1 because the sunspot data has not been normalised.

Fig. 1. Monthly observations of sunspot numbers [1].

and is parallel to the membership interval at the point of intersection.

Definition 5 (Lower Membership Grade). The Lower Membership Grade (LMG) of a vertical slice of an interval-valued complex fuzzy set is the lower bound of its membership interval.

Definition 6 (Upper Membership Grade). The Upper Membership Grade (UMG) of a vertical slice of an interval-valued complex fuzzy set is the upper bound of its membership interval.

\section{B. The Interval-Valued Complex FIS}

An interval-valued complex FIS is a hybrid of a complex FIS and an interval-valued FIS. An interval-valued FIS is 
equivalent to an interval type-2 FIS. It would be unsurprising if the interval-valued complex FIS were to inherit properties from both the complex FIS and the interval type-2 FIS. Figure 2 shows the various stages of the interval-valued complex FIS.

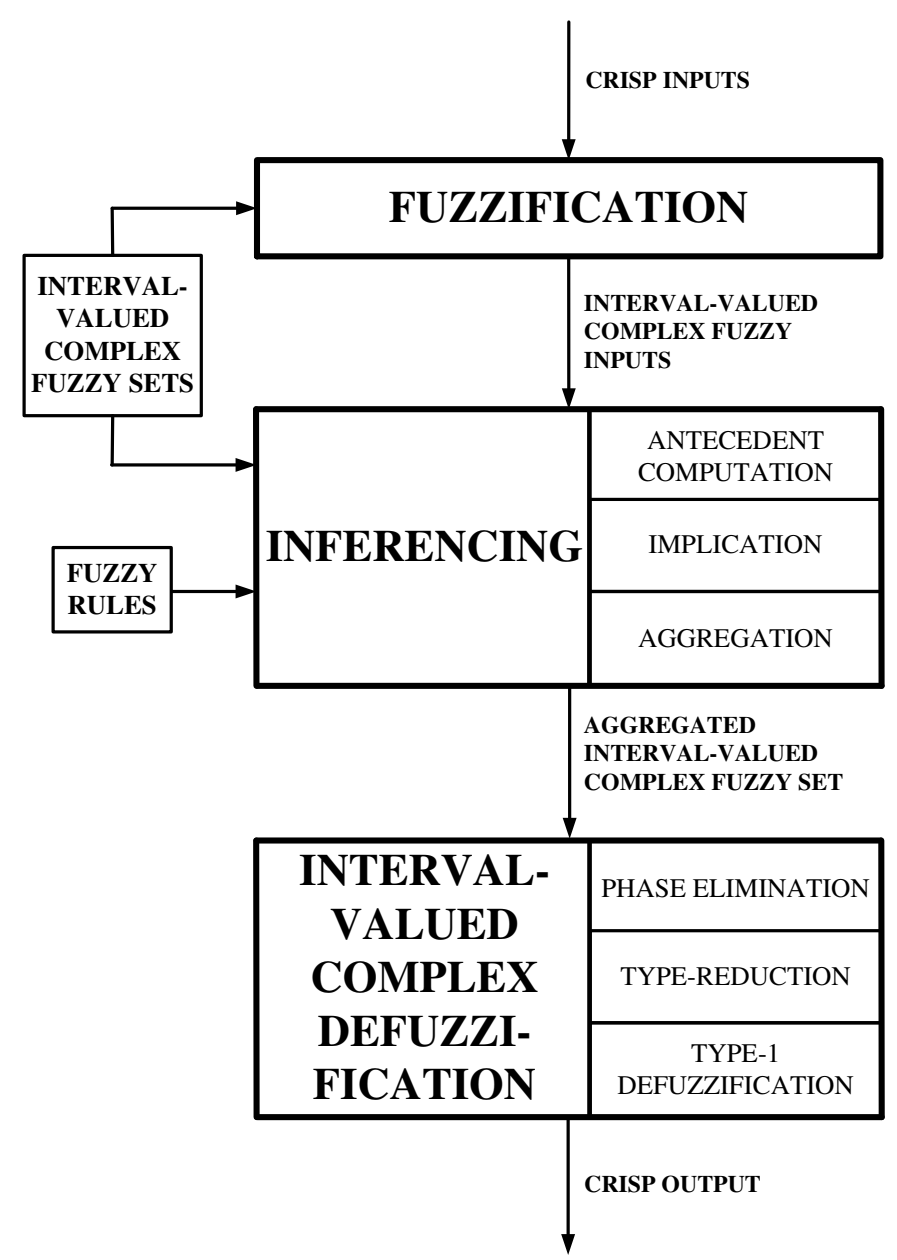

Fig. 2. The interval-valued complex Mamdani FIS [9].

\section{Rotational Invariance}

Ramot et al. point out that phase is relative, and that therefore the absolute value of the phase term is inconsequential:

"Due to the relative nature of phase, the absolute value of the phase term is often of little significance. In fact, the absolute value of $\omega_{S}(x)$ is usually determined according to some arbitrary reference, which may vary from one application to another. Therefore, neither a value of $\pi$ nor zero may be considered as a high or low value of membership phase. Instead, the important parameter is dominantly the relative phase between $x$ and other elements in the support of $S . "$ [22, page 173]

Based on this observation, Dick characterises the 'intuitively appealing property' of rotational invariance:
"The assertion that "phase is purely relative" can be taken to mean that a uniform phase shift in the arguments of a union, intersection or complement of complex fuzzy sets should induce the same uniform phase shift in the result of these operations. Thus, if we rotate two vectors about the origin by a common value (say $\varphi$ ), a union or intersection of those vectors will be rotated by the same value." [5, page 409]

Definition 7 (Rotational Invariance [5]). Let $\mathrm{D}$ be the unit disc $D \subset \mathbb{R} \times \mathbb{R}$. A function $F: \mathbf{D} \times \mathbf{D} \rightarrow \mathbf{D}$ is rotationally invariant iff

$$
F\left(x e^{j \alpha} \cdot e^{j k}, y e^{j \beta} \cdot e^{j k}\right)=e^{j k} \cdot F\left(x e^{j \alpha}, y e^{j \beta}\right)
$$

for all elements $x e^{j \alpha}, y e^{j \beta} \in \mathbf{D}$.

\section{INTERVAL-VALUED COMPLEX JOIN AND MEET}

\section{A. Complex Union and Intersection}

The fuzzification stage, and the antecedent combination and implication substages of the inferencing stage are driven by union and intersection operations in the type- 1 case. Ramot et al. [21, page 452] extend these operations to complex fuzzy sets. Union is defined thus:

"Let $\mu_{A}(x)=r_{A}(x) \cdot e^{j \omega_{A}(x)}$ and $\mu_{B}(x)=r_{B}(x)$. $e^{j \omega_{B}(x)}$. Then, the membership function of $A \cup B$ is given by

$$
\mu_{A \cup B}(x)=\left[r_{A}(x) \oplus r_{A}(x)\right] \cdot e^{j \omega_{A \cup B}(x)}
$$

where $\oplus$ represents a t-conorm function."

Suggested techniques for calculating $\omega_{A \cup B}$ are [21, page 452]:

1) Sum: $\omega_{A \cup B}=\omega_{A}+\omega_{B}$;

2) Maximum: $\omega_{A \cup B}=\max \left(\omega_{A}, \omega_{B}\right)$;

3) Minimum: $\omega_{A \cup B}=\min \left(\omega_{A}, \omega_{B}\right)$;

4) Winner Takes All: $\omega_{A \cup B}=\left\{\begin{array}{l}\omega_{A}, r_{A}>r_{B} \\ \omega_{B}, r_{B}>r_{A}\end{array}\right.$

Options 2, 3 and 4 are rotationally invariant. Intersection is defined analogously to union (using a t-norm instead of a tconorm). Suggested methods for calculating $A \cap B$ are the same as those for calculating $A \cup B$ [21, page 453].

\section{B. Interval-Valued Complex Join and Meet}

The definitions of complex union and intersection may be extended to interval-valued complex join and meet. Considering join first, the t-conorm in Equation 1 for complex union may be replaced by the interval type- 2 join operation [19]. Of the techniques suggested for finding $A \cup B$, the first three are independent of the modulus, so may be employed for interval-valued complex join without adaptation. The fourth technique includes moduli among the parameters. These are crisp numbers, as opposed to intervals. The intervals in the interval-valued complex membership functions need to be reduced to crisp numbers in order for the fourth technique to be applicable, which can easily be achieved by taking the mid-point of the interval as a representative value. Then the definitions of complex union may be applied, with the moduli 
replaced by the interval mid-points as a representative value, though any method of ordering interval numbers [4] would be a valid alternative. The meet operation may be defined analogously to join.

The four techniques for calculating $A \cup B$ together with the four corresponding strategies for calculating $A \cap B$, give rise to eight algorithms (Algorithms $1-8$ ). In Algorithms $2-8$, lines $1-7$ are the same as in Algorithm 1, so have not been stated explicitly.

As they are based on Options 2, 3 and 4 of the suggested complex union and intersection techniques (Subsection III-A), Algorithms 3 to 8 possess the important property of rotational invariance.

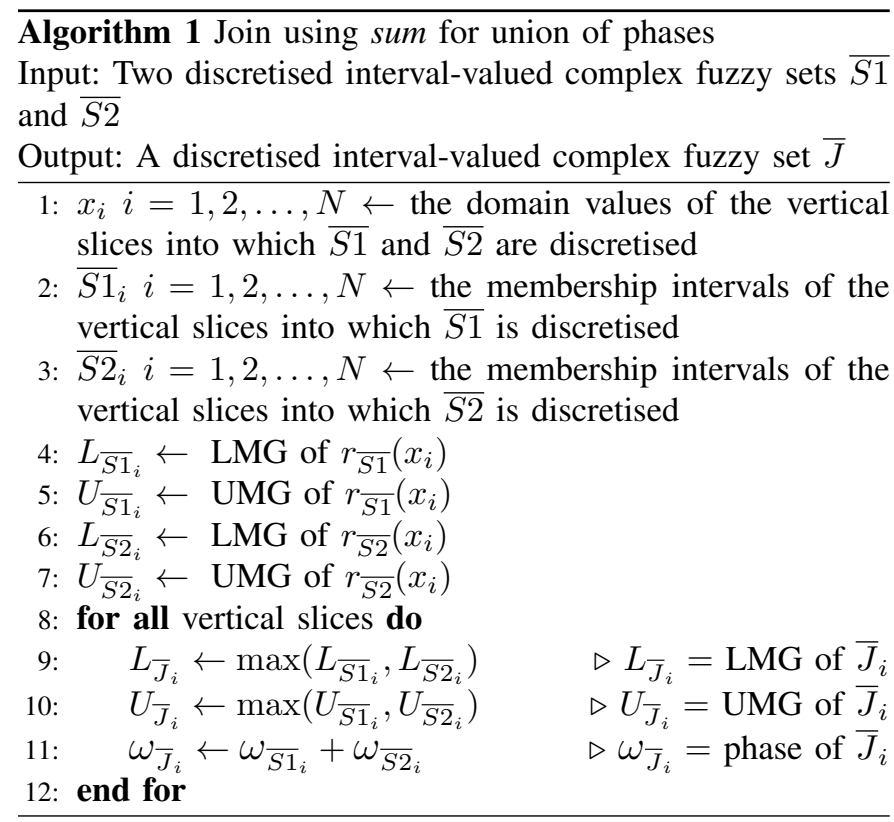

Algorithm 2 Meet using sum for intersection of phases Input: Two discretised interval-valued complex fuzzy sets $\overline{S 1}$ and $\overline{S 2}$

Output: A discretised interval-valued complex fuzzy set $\bar{M}$

\begin{tabular}{cc}
\hline 8: for all vertical slices do & \\
9: $\quad L_{\bar{M}_{i}} \leftarrow \min \left(L_{\overline{S 1}_{i}}, L_{\overline{S 2}_{i}}\right)$ & $\triangleright L_{\bar{M}_{i}}=$ LMG of $\bar{M}_{i}$ \\
10: $\quad U_{\bar{M}_{i}} \leftarrow \min \left(U_{\overline{S 1}_{i}}, U_{\overline{S 2}_{i}}\right)$ & $\triangleright U_{\bar{M}_{i}}=$ UMG of $\bar{M}_{i}$ \\
11: $\quad \omega_{\bar{M}_{i}} \leftarrow \omega_{\overline{S 1}_{i}}+\omega_{\overline{S 2}_{i}}$ & $\triangleright \omega_{\bar{M}_{i}}=$ phase of $\bar{M}_{i}$ \\
12: end for & \\
\hline
\end{tabular}

\begin{tabular}{|c|c|}
\hline \multirow{2}{*}{\multicolumn{2}{|c|}{$\begin{array}{l}\text { Algorithm } 3 \text { Join using maximum for union of phases } \\
\text { Input: Two discretised interval-valued complex fuzzy sets } \bar{S} \\
\text { and } \bar{S} 2 \\
\text { Output: A discretised interval-valued complex fuzzy set } \bar{J}\end{array}$}} \\
\hline & \\
\hline 8: for all vertical slices do & \\
\hline$L_{\bar{J}_{i}} \leftarrow \max \left(L_{\overline{S 1}_{i}}, L_{\overline{S 2}_{i}}\right)$ & $L_{\bar{J}_{i}}=\mathrm{LMG}$ of \\
\hline$U_{\bar{J}_{i}} \leftarrow \max \left(U_{\overline{S 1}_{i}}, U_{\overline{S 2}_{i}}\right)$ & $U_{\bar{J}_{i}}=\mathrm{UMG}$ of. \\
\hline$\omega_{\bar{J}_{i}} \leftarrow \max \left(\omega_{\overline{S 1}_{i}}, \omega_{\overline{S 2}_{i}}\right)$ & $\omega_{\bar{J}_{i}}=$ phase \\
\hline
\end{tabular}

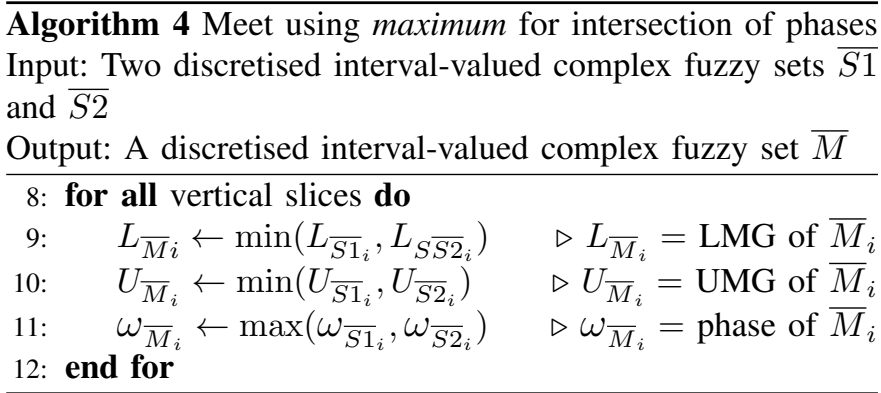

Algorithm 5 Join using minimum for union of phases Input: Two discretised interval-valued complex fuzzy sets $\overline{S 1}$ and $\overline{S 2}$

Output: A discretised interval-valued complex fuzzy set $\bar{J}$

\begin{tabular}{ccc}
\hline 8: & for all vertical slices do & \\
9: $\quad L_{\bar{J}_{i}} \leftarrow \max \left(L_{\overline{S 1}_{i}}, L \overline{S 2}_{i}\right)$ & $\triangleright L_{\bar{J}_{i}}=$ LMG of $\bar{J}_{i}$ \\
10: $\quad U_{\bar{J}_{i}} \leftarrow \max \left(U_{\overline{S 1}_{i}}, U_{\overline{S 2}_{i}}\right)$ & $\triangleright U_{\bar{J}_{i}}=$ UMG of $\bar{J}_{i}$ \\
11: $\quad \omega_{\bar{J}_{i}} \leftarrow \min \left(\omega_{\overline{S 1}_{i}}, \omega_{\overline{S 2}_{i}}\right)$ & $\triangleright \omega_{\bar{J}_{i}}=$ phase of $\bar{J}_{i}$ \\
12: end for & \\
\hline
\end{tabular}

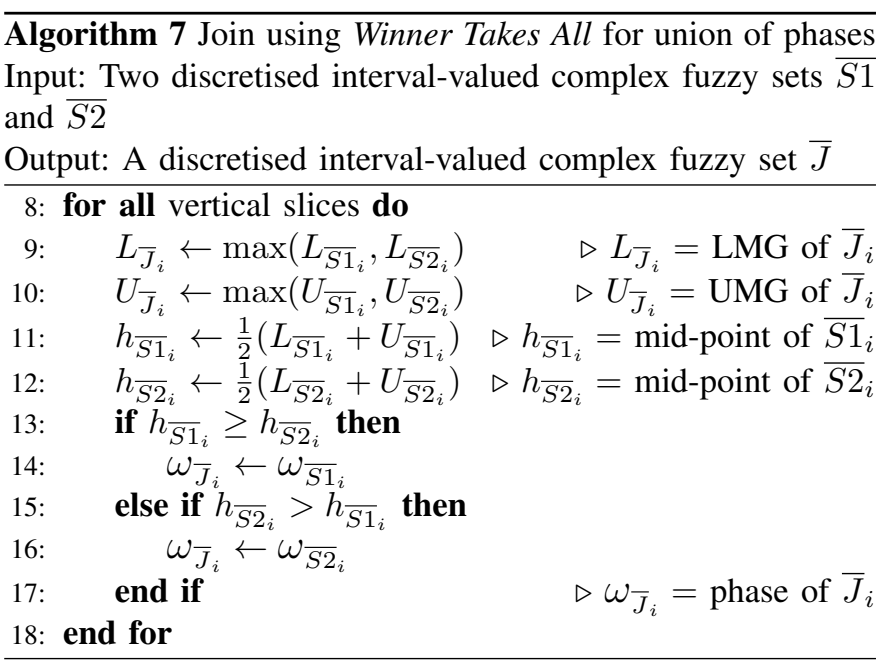

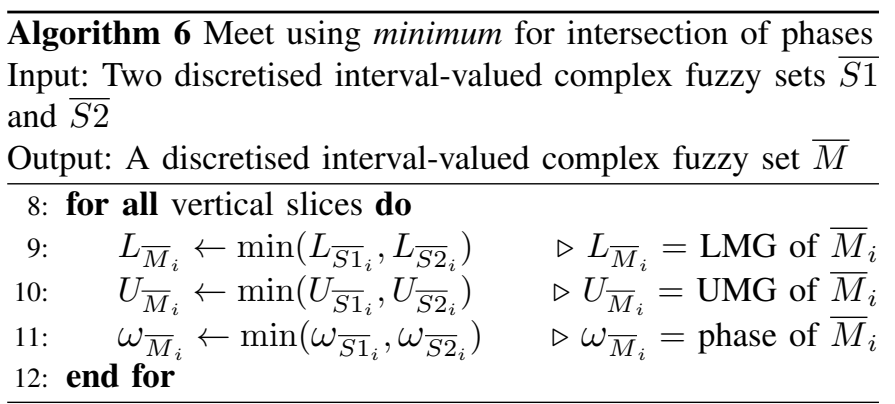




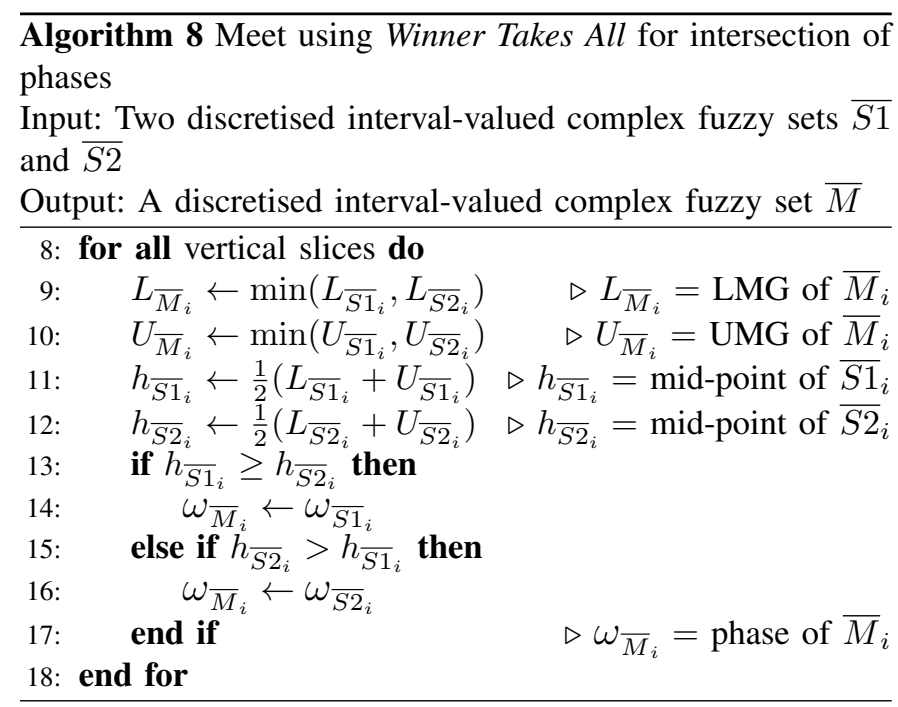

\section{CONCLUSION}

This investigation presented a selection of algorithms for the join and meet operations of interval-valued complex fuzzy logic. It was shown that six out of the eight algorithms documented possess the intuitive and significant property of rotational invariance.

\section{A. Further Work}

1) Lattice-Theoretic Analysis: The understanding of interval-valued complex join and meet operations would be advanced by investigating whether the algorithms presented here form a complete, distributed lattice over $[0,1]$.

2) Prototype Interval-Valued Complex FIS: The development of a prototype interval-valued complex FIS would be welcome, dealing with uncertain inputs where either seasonality is a consideration. Its performance could then be contrasted experimentally with a non-interval complex FIS and a conventional interval type-2 FIS.

3) Generalised Type-2 Complex FIS: Following naturally from the work reported in this paper would be research into generalised type- 2 complex fuzzy join and meet operations.

4) Aggregation Operators: There is some communality between join and meet operators and aggregation operators. In [9], the subject of aggregation operators for the IVCFIS was touched upon, but more research is necessary to specify suitable algorithms.

5) Restriction of Phases to Principal Values: Amending the definition of rotational invariance (Subsection II-C) so that phases are restricted to principal values has implications, most notably that Options 2 and 3 for union and intersection (Subsection III-A) would cease to be rotationally invariant. The impact the principal values restriction would have on the IVCFIS as a whole is a topic warranting further investigation.

\section{REFERENCES}

[1] Australian Government Bureau of Meteorology: Radio and Space Weather Services. Monthly Sunspot Numbers, 2013. http://www.ips. gov.au/Solar/1/6 [Online; accessed 21-February-2013].
[2] Nora Boumella, Karim Djouani, and Mohamed Boulemden. On an Interval Type-2 TSK FLS A1-C1 Consequent Parameters Tuning. In Proceedings of the IEEE Symposium on Advances in Type-2 Fuzzy Logic Systems 2011, Paris, April 2011.

[3] Humberto Bustince. Interval-valued fuzzy sets in soft computing. International Journal of Computational Intelligence Systems, 3(2):215 $-222,2010$.

[4] Joaquín Derrac, Francisco Chiclana, Salvador García, and Francisco Herrera. Evolutionary fuzzy k-nearest neighbors algorithm using intervalvalued fuzzy sets. Information Sciences, 329:144 - 163, 2016.

[5] Scott Dick. Toward Complex Fuzzy Logic. IEEE Transactions on Fuzzy Systems, 13(3):405-414, June 2005. DOI: http://dx.doi.org/10.1109/TFUZZ.2004.839669.

[6] Mikel Galar, Edurne Barrenechea, Javier Fernandez, Humberto Bustince, and Gleb Beliakov. Representing Images by Means of Interval-Valued Fuzzy Sets. Application to Stereo Matching. In Proceedings of the IEEE Symposium on Advances in Type-2 Fuzzy Logic Systems 2011, Paris, April 2011.

[7] Sarah Greenfield. Uncertainty Measurement for the Interval Type-2 Fuzzy Set. Accepted for the 15th International Conference on Artificial Intelligence and Soft Computing (ICAISC 2016), Zakopane, Poland.

[8] Sarah Greenfield and Francisco Chiclana. Fuzzy in 3D: Two Contrasting Paradigms. Archives for the Philosophy and History of Soft Computing, 2, 2015. http://www.aphsc.org/index.php/aphsc/article/view/24 [Online; accessed 20-January-2016].

[9] Sarah Greenfield, Francisco Chiclana, and Scott Dick. Interval-Valued Complex Fuzzy Logic. Accepted for FUZZ-IEEE 2016, Vancouver, Canada.

[10] Sarah Greenfield and Robert I. John. The Uncertainty Associated with a Type-2 Fuzzy Set. In Rudolf Seising (editor) Views on Fuzzy Sets and Systems from Different Perspectives, in 'Studies in Fuzziness and Soft Computing', series editor Janusz Kacprzyk, volume 243, pages 471483. Springer-Verlag, 2009. DOI: http://dx.doi.org/10.1007/978-3-54093802-6_23.

[11] Hani Hagras and Christian Wagner. Introduction to Interval Type2 Fuzzy Logic Controllers - Towards Better Uncertainty Handling in Real World Applications. IEEE Systems, Man and Cybernetics eNewsletter, 2009. Issue 27.

[12] Robert I. John and Simon Coupland. Type-2 Fuzzy Logic: A Historical View. IEEE Computational Intelligence Magazine, 2(1):57 - 62, February 2007. DOI: 10.1109/MCI.2007.357194.

[13] Aranzazu Jurio, Daniel Paternain, Carlos Lopez-Molina, Humberto Bustince, Radko Mesiar, and Gleb Beliakov. A Construction Method of Interval-Valued Fuzzy Sets for Image Processing. In Proceedings of the IEEE Symposium on Advances in Type-2 Fuzzy Logic Systems 2011, Paris, April 2011

[14] Erdal Kayacan, Ozkan Cigdem, and Okyay Kaynak. On Novel Training Method Based on Variable Structure Systems Approach for Interval Type-2 Fuzzy Neural Networks. In Proceedings of the IEEE Symposium on Advances in Type-2 Fuzzy Logic Systems 2011, Paris, April 2011.

[15] Leonardo Leottau and Miguel Melgarejo. Implementing an Interval Type-2 Fuzzy Processor onto a DSC 56F8013. In Proceedings of FUZZIEEE 2010, pages 1939-1942, Barcelona, Spain, 2010.

[16] Chunshien Li and Tai-Wei Chiang. Intelligent financial time series forecasting: A complex neuro-fuzzy approach with multi-swarm intelligence. International Journal of Applied Mathematics and Computer Science, 22(4):787 - 800, 2012.

[17] Ondrej Linda and Milos Manic. Uncertainty Modelling for Interval Type-2 Fuzzy Logic Systems Based on Sensor Characteristics. In Proceedings of the IEEE Symposium on Advances in Type-2 Fuzzy Logic Systems 2011, Paris, April 2011.

[18] Jun Ma, Guangquan Zhang, and Jie Lu. A Method for Multiple Periodic Factor Prediction Problems Using Complex Fuzzy Sets. IEEE Transactions on Fuzzy Systems, 20(1):32 - 45, 2012. DOI: http://dx.doi.org/10.1109/TFUZZ.2011.2164084

[19] Jerry M. Mendel. Uncertain Rule-Based Fuzzy Logic Systems: Introduction and New Directions. Prentice-Hall PTR, 2001.

[20] Jerry M. Mendel and Robert I. John. Type-2 fuzzy sets made simple. IEEE Transactions on Fuzzy Systems, 10(2):117 - 127, 2002. DOI: http://dx.doi.org/10.1109/91.995115.

[21] Daniel Ramot, Menahem Friedman, Gideon Langholz, and Abraham Kandel. Complex Fuzzy Logic. IEEE Transactions on Fuzzy Systems, 11(4):450 - 461, 2003. DOI: http://dx.doi.org/10.1109/TFUZZ.2003.814832. 
[22] Daniel Ramot, Ron Milo, Menahem Friedman, and Abraham Kandel. Complex Fuzzy Sets. IEEE Transactions on Fuzzy Systems, 10(2):171 - 186, 2002. DOI: http://dx.doi.org/10.1109/91.995119.

[23] José A. Sanz, Alberto Fernández, Humberto Bustince, and Francisco Herrera. A Genetic Tuning to Improve the Performance of Fuzzy RuleBased Classification Systems with Interval-Valued Fuzzy Sets: Degree of Ignorance and Lateral Position. International Journal of Approximate Reasoning, 52:751-766, 2011.

[24] Arturo Tellez-Velazquez, Heron Molina-Lozano, Marco A. MorenoArmendariz, Elsa Rubio-Espino, Luis A. Villa-Vargas, and Ildar Batyrshin. Parametric Type-2 Fuzzy Control Design for the Ball and Plate System. In Proceedings of the IEEE Symposium on Advances in Type-2 Fuzzy Logic Systems 2011, Paris, April 2011.

[25] Omolbanin Yazdanbakhsh and Scott Dick. Multi-Variate Timeseries Forecasting Using Complex Fuzzy Logic. In Proceedings of NAFIPS 2015, pages $1-6$, Redmond, Washington, 2015.

[26] Omolbanin Yazdanbakhsh, Alix Krahn, and Scott Dick. Predicting solar power output using complex fuzzy logic. In Proceedings of IFSA/NAFIPS 2013, pages 1243 - 1248, Edmonton, Alberta, Canada, 2013.

[27] Lotfi A. Zadeh. Fuzzy Sets. Information and Control, 8:338 - 353, 1965.

[28] Lotfi A. Zadeh. The Concept of a Linguistic Variable and its Application to Approximate Reasoning. Information Sciences, 8:199 - 249, 1975.

[29] Lotfi A. Zadeh. The Concept of a Linguistic Variable and its Application to Approximate Reasoning - II. Information Sciences, 8:301 - 357, 1975.

[30] Lotfi A. Zadeh. The Concept of a Linguistic Variable and its Application to Approximate Reasoning - III. Information Sciences, 9:43 - 80, 1975. 